\title{
Identification of MAP3K15 as a Potential Prognostic Biomarker and Correlation with Immune Infiltrates in Osteosarcoma
}

\section{Zhuning Chen}

Sun Yat-Sen University

Haoran Kong

Sun Yat-Sen University

Zhaopeng Cai

Sun Yat-Sen University

Keng Chen

Sun Yat-Sen University

\section{Boyang Wu}

Sun Yat-Sen University

Haonan Li

Sun Yat-Sen University

Peng Wang

Sun Yat-Sen University

Yanfeng Wu

Sun Yat-Sen University

Huiyong Shen ( $\square$ shenhuiy@mail.sysu.edu.cn )

Sun Yat-Sen University https://orcid.org/0000-0002-4317-3468

\section{Research Article}

Keywords: MAP3K15, immune infiltrates, osteosarcoma, prognosis, bioinformatics

Posted Date: April 20th, 2021

DOI: https://doi.org/10.21203/rs.3.rs-416934/v1

License: (c) (1) This work is licensed under a Creative Commons Attribution 4.0 International License.

Read Full License 


\section{Abstract}

Background: Osteosarcoma (OS) is a type of primary malignant tumor, and increasing amounts of evidence show the clinical benefits of immunotherapy in treating OS. However, the lack of comprehensive studies on the complex OS immune microenvironment hinders the application of immunotherapy. Thus, we aimed to systematically study the immune characteristics of OS and identify novel biomarkers for OS treatment.

Methods: We systematically studied the immune score and proportion of infiltrating immune cells in OS in the Therapeutically Applicable Research to Generate Effective Treatments (TARGET) and Gene Expression Omnibus (GEO) databases using the ESTIMATE and CIBERSORT algorithms. Differential expression and functional analyses were used to identify dysregulated genes and explore their functions. Survival and Cox regression analyses were applied to establish an immune-related prognostic signature. The expression of MAP3K15 was performed by qPCR assay and immunohistochemistry. We divided 48 patients into high expression and low expression groups according to MAP3K15 expression.

Results: A total of 103 differentially expressed immune genes (DEIGs) were found in the TARGET-OS and GSE39058 databases, and these DEIGs were mainly enriched in leukocyte proliferation, leukocyte differentiation, osteoclast differentiation, natural killer (NK) cell-mediated cytotoxicity and the adaptive immune system. A predictive signature was constructed based on the survival analysis, with an area under the receiver operating characteristic (ROC) curve (AUC) of 0.65 . Moreover, we found that mitogenactivated protein kinase kinase kinase 15 (MAP3K15) can predict the prognosis of patients with OS and is closely related to CD4 T cells, M0 macrophages, and M1 macrophages. We found that patients with high MAP3K15 expression had a significantly poorer prognosis

Conclusions: Our study found that MAP3K15, whose expression level is closely related to immune activity in tumors, was a critical immune-related biomarker and may provide a basis for OS immunotherapy.

\section{Clinical Trial Registration}

Not applicable.

\section{Background}

Osteosarcoma (OS) is a type of primary malignant tumor that commonly occurs in children and adolescents [1]. Although its incidence is not high, OS easily relapses and metastasizes and has a fiveyear survival rate of approximately $65 \%[1,2]$. Currently, the treatment for OS mainly consists of three parts: preoperative chemotherapy, surgical resection, and postoperative radiotherapy [3, 4]. Surgical resection is still the primary treatment for OS [3]. However, traditional medicines are unsatisfactory in reducing the recurrence and metastasis of OS $[5,6]$. Recently, immunotherapy has made breakthroughs in the field of cancer therapy. Immune checkpoint inhibitors, such as PD-1, PD-L1, and CTAL4, have shown significant antitumor effects on various cancers [7-9]. Additionally, these immune checkpoint inhibitors 
have been elucidated to play substantial roles in the treatment of OS. However, immunotherapy is still not beneficial to many patients because of target mismatch and drug resistance $[10,11]$. Thus, there is an urgent need to identify novel immune-related biomarkers to better guide clinical immunotherapy for OS.

The tumor microenvironment (TME) is the cellular environment that includes endothelial cells, mesenchymal cells, immune cells, inflammatory mediators, and extracellular matrix molecules [12-14]. Further studies on the complexity and systemic characteristics of the TME are greatly needed to identify useful biomarkers. Based on the ESTIMATE and CIBERSORT algorithms, the gene expression matrix data could be converted into an immune score and a proportion of infiltrating immune cells, which could be used to study the immune activity of the TME [15-17]. With the help of these two algorithms, many studies have explored immunity in the TME of lung cancer [18], breast cancer [19], and liver cancer [20]. These studies provide direction for tumor immunotherapy by exploring the immune-related genes and immune cells that play crucial roles in the TME.

In this study, we collected the expression profiles and clinical information of OS patients using the public database Therapeutically Applicable Research to Generate Effective Treatments (TARGET) and the GSE39058 dataset in the Gene Expression Omnibus (GEO) database. First, the ESTIMATE algorithm was used to identify the significant prognostic value of immune infiltration in the TME. To investigate the useful immune-related biomarkers in OS, we performed difference analysis and functional enrichment analysis and constructed a protein-protein interaction (PPI) network for the two datasets. After Cox regression analysis, mitogen-activated protein 3 kinase 15 (MAP3K15) was considered to play an important role in the immune microenvironment. Subsequently, the proportion of infiltrating immune cells was obtained by the CIBERSORT algorithm to further explore the correlation between MAP3K15 expression and the score of immune cells. The analysis showed that MAP3K15 had the most significant relationship with CD4 T cells, M0 macrophages, and M1 macrophages. In summary, this study found that MAP3K15, whose expression level is closely related to immune activity in tumors, was a critical immunerelated biomarker and may provide a basis for OS immunotherapy.

\section{Methods}

\subsection{Samples}

Forty-eight osteosarcoma tissues were obtained from patients at Sun Yat-sen Memorial Hospital of Sun Yat-sen University and The Eighth Affiliated Hospital of Sun Yat-sen University and between December 2010 and March 2018. None of the patients underwent chemotherapy or radiotherapy before surgery. The tissues were immediately snap-frozen and stored at $-80^{\circ} \mathrm{C}$ in an ultralow temperature freezer until further analysis. The study was approved by the Ethics Committee of Sun Yat-sen Memorial Hospital of Sun Yatsen University and The Eighth Affiliated Hospital of Sun Yat-sen University. Informed consent forms were signed by all individuals prior to acquiring and studying their tissues.

\subsection{Obtaining Expression Data from Publicly Available Data}


The OS primary data were downloaded from the TARGET database and GEO database. Accession number GSE39058 was chosen in GEO for data analysis, and it contained a total of 164 samples, of which 46 included mRNA profiling data. Additionally, clinical outcomes were included in the dataset. Then, the microarray data of mRNA expression were normalized via a log2 scale transformation, and the average expression was used when a gene was detected with more than one probe. In addition, the RNAseq data and relevant clinical information were downloaded from the TARGET database (https://ocg.cancer.gov/programmes/target). Both fragments per kilobase of transcript per million mapped reads (FPKM) and counts were used for further data processing. This study followed the publication guidelines of the GEO and TARGET databases.

\subsection{Identification of the Tumor Microenvironment and Infiltrating Immune Cells}

First, the ESTIMATE algorithm [15] was performed to identify the immune score and stromal score. According to the median values of the stromal/immune scores, we divided OS patients into a high-risk group and a low-risk group. Then, Kaplan-Meier survival curves were used to analyze the correlation between the clinical outcomes and the stromal/immune scores.

Additionally, the characterization of the cellular composition of OS was analyzed based on the CIBERSORT algorithm [17]. A heatmap was used to visualize the proportion of immune cells in the lowand high-risk immune score groups. In addition, we further applied Spearman's correlation coefficient to verify the relationship between immune/stromal scores and immune cells. Both the ESTIMATE and CIBERSORT algorithms were performed based on R 4.0.2 and RStudio (version 1.2.5001).

\subsection{Differential Expression Analysis and Functional Analysis}

The Limma R package [21] was applied to identify the differentially expressed immune genes (DEIGs) between the low and high immune score groups. Then, the common DEIGs were obtained from GSE39058 and TARGET-OS. Here, $\mid \log _{2} \mathrm{FCl} \geq 1$ and $\mathrm{P}<0.05$ were set as the cutoff values.

Furthermore, to explore the potential functions of these DEIGs, Gene Ontology (GO) and Kyoto Encyclopedia of Genes and Genomes (KEGG) analyses were performed via the Metascape database (http://metascape.org). Additionally, overexpressed and underexpressed immune gene groups were input, and the PPI network and molecular complex detection (MCODE) were used to further identify the densely connected regions. $\mathrm{P}<0.05$ was used as a cutoff value.

\subsection{Construction of the Prognostic Immune Gene Signatures}

TARGET-OS and GSE39058 were used to establish a prognostic immune gene signature, with TARGET-OS being the training set and GSE39058 being the validation set. Univariate Cox regression analysis was first performed to identify survival-related immune genes via the SangerBox database (http://sangerbox.com/). Additionally, a robust likelihood-based survival analysis was used to increase 
the reliability and accuracy, allowing for iterative forward selection of multiple sets of genes based on the partial likelihood of the Cox model.

Then, multivariate Cox regression was implemented to explore the independent prognostic predictor genes. Additionally, the survival receiver operating characteristic (ROC) curve was obtained from SangerBox, and then the area under the ROC curve (AUC) was used to assess the efficiency of the predictive signature. $\mathrm{P}<0.05$ was considered statistically significant.

\subsection{Correlation Analysis Between Immune Cells and the Prognostic Signature}

The proportion of 22 infiltrating immune cells was obtained according to the CIBERSORT algorithm. The Wilcoxon signed-rank test (two groups) or Kruskal-Wallis rank sum test (more than two groups) were conducted to calculate the correlation between immune cells and clinical characteristics. Additionally, a log-rank test was used to evaluate the association between immune cells and the prognostic signature. $P$ $<0.05$ was regarded as statistically significant.

\subsection{Quantitative Polymerase Chain Reaction (qPCR) Assay}

Extraction of total RNA was performed with TRIzol reagent (Life Technologies) following the manufacturer's instructions; the extracted RNA was then quantified. Next, qPCR was performed using a BeyoFast $^{\text {TM }}$ SYBR Green One-Step qRT-PCR Kit (Beyotime, D7268S). The expression of MAP3K15was normalized to that of GAPDH. Relative expression levels were calculated with the $2-\triangle \triangle \mathrm{Ct}$ method.

\subsection{Immunohistochemistry Validation}

Immunohistochemistry (IHC) was performed according to the antibody supplier instructions. The slices of the clinical samples were incubated with primary antibodies at different dilution ratios against MAP3K15 overnight at $4^{\circ} \mathrm{C}$. Images were captured at an appropriate magnification under a microscope (Nikon Microsystems, Shanghai, China). The antibody used in the study was anti-MAP3K15 (SAB, \#45534).

\section{Results}

\subsection{Immune and Stromal Scores were Evaluated by the ESTIMATE Algorithm}

First, 86 OS patients with gene expression profiles and clinical characteristics were identified in the TARGET-OS dataset. The ESTIMATE algorithm was then performed to calculate the immune and stromal scores of tumor tissues. Depending on the calculations, immune scores were distributed between 1842.4 and 2013.6, while stromal scores ranged from - 2124.1 to 3901.0. To explore the prognostic significance of immune and stromal scores, we divided the OS patients into high and low immune score groups according to their scores. As shown in Kaplan-Meier curves, although the estimated group was not statistically significant $(P=0.05)$, the stromal group $(P=0.014)$ and immune group $(P=0.0019)$ were 
positively correlated with prognosis, indicating that both were positive factors in the prognosis of OS patients (Fig. 1a-c).

Additionally, the same processes were performed on the GSE39058 dataset. According to the ESTIMATE calculation, the immune scores ranged from -1116.8 to 1676.5 , while stromal scores were between 2677.4 and 2916.059471. However, when the groups were divided into high and low immune score groups according to their scores, the results showed that there was no statistically significant difference in the prognosis between the immune group $(P=0.23)$ and the stromal group $(P=0.84)$ (Fig. 1d-f).

\subsection{Differentially Expressed Immune Genes and Functional Analysis}

To explore the correlation between immune genes and immune scores, we compared 46 OS patients in the GSE39058 dataset and 86 patients in the TARGET-OS dataset. As shown in Fig. 2a-c, a total of 1446 DEIGs were discovered in GSE39058, including 729 genes that were upregulated and 735 that were downregulated. In addition, a total of 565 DEIGs were identified in the TARGET-OS dataset, with 502 genes that were upregulated and 63 genes that were downregulated. Then, 100 commonly upregulated genes and 3 commonly downregulated genes were extracted via a Venn diagram.

Subsequently, as shown in Fig. $2 \mathrm{~d}$ and e, GO enrichment analysis indicated that the common DEIGs were enriched in various immune processes, such as leukocyte proliferation, regulation of leukocyte differentiation, negative regulation of leukocyte activation, regulation of innate immune response and leukocyte-mediated cytotoxicity, while KEGG pathway analysis suggested that osteoclast differentiation, natural killer (NK) cell-mediated cytotoxicity and adaptive immune system were the most pivotal pathways affected by these genes. Thus, these dysregulated immune genes might be the basis of the changes in the TME and enhance the mechanism of immune escape.

Furthermore, the PPI network and MCODE plugin based on the Metascape database identified the significant modules in these DEIGs. Module 1 included 33 edges and 9 nodes and involved MAP3K15, CDC247, and HLA-DRB1. Module 2 included 6 edges and 4 nodes and involved CCL23, CCL5, GPR55, and LNP3 (Fig. 2f).

\subsection{Identification of a Prognosis-related Gene}

Since 103 common DEIGs were obtained from the previous step, univariate Cox regression analysis was first performed to identify the survival-related genes. According to the median expression level of immune genes, OS patients were divided into high and low expression groups. As shown in Figs. 3, 3 and 12 genes were identified to be correlated with prognosis in the GSE39058 and TARGET-OS datasets, respectively. Additionally, Kaplan-Meier curves were drawn to visualize the results.

Then, we noticed that only MAP3K15 was common in the results from the two datasets. Thus, multivariate Cox regression was implemented to explore the independent prognostic value of MAP3K15. As shown in Fig. 4, MAP3K15 was verified to be statistically significant. Moreover, ROC curves were used 
to determine the efficiency of the Cox regression, and the 5-year AUC was 0.65. In addition, the result was verified in GSE39058, with an AUC of 0.63 (Supplementary Fig. 1). Therefore, the expression of MAP3K15 could predict OS patients' clinical outcomes and may provide novel insights into the changes in the TME and the mechanism of immune escape.

\subsection{Immune Landscapes Evaluated by CIBERSORT}

There are a considerable number of tumor cells and immune cells in the tumor environment.

Understanding the proportion of immune landscapes of the tumor may reveal the underlying mechanism of refractory tumors. Thus, the CIBERSORT algorithm was applied to explore the proportion of immune cells in the GSE39058 and TARGET-OS datasets based on the gene expression data. Here, analysis of the GSE39058 and TARGET-OS datasets was processed separately. Although the proportion of immune cells suggested different immune landscapes in the two datasets, CD4 naive T cells, CD4 memory resting T cells, M0 macrophages, M2 macrophages, and naive B cells accounted for a large proportion of infiltrating immune cells in OS (Fig. $5 \mathrm{a}$ and b). Moreover, violin plots were used to visualize the immune cell subsets between the high and low immune score groups (Fig. $5 \mathrm{c}$ and d), and the results showed that the proportion of memory B cells, naive T cells, M2 macrophages and activated NK cells was significantly increased.

Additionally, to investigate the rationality of our predictive signature, we further calculated the proportion of immune cells in the high- and low-risk immune score groups (Fig. 5e and f). The results demonstrated that the proportion of CD4 T cells, M1 macrophages and M2 macrophages was significantly increased. Thus, the differences between the high- and low-risk score groups may provide clues to the mechanism of immune microenvironment regulation.

\subsection{Correlations between the Prognostic Signature and Infiltrating Immune Cells}

Since the results above revealed that MAP3K15 might play important roles in the TME, we wondered about the connection between MAP3K15 and the relevant immune cells. According to the CIBERSORT algorithm, a correlation between MAP3K15 and immune cells was found. As shown in Fig. 6a-c, the expression of MAP3K15 and CD4 T cells, M0 macrophages, and M1 macrophages was positively correlated.

Furthermore, according to the ESTIMATE algorithm used, we found that MAP3K15 was positively correlated with the immune score, which further suggested the significance of MAP3K15 in the TME (Fig. 6d).

\subsection{Validation}

To verify the results obtained by bioinformatics analysis, we performed a qPCR assay of MAP3K15 expression in osteosarcoma tissues. We divided 48 patients into high expression and low expression groups according to MAP3K15 expression and found that patients with high MAP3K15 expression had a 
significantly poorer prognosis (Fig. 7a). Moreover, the immunohistochemistry results showed that the expression trend of MAP3K15 protein was the same as that of mRNA (Fig. 7b). Hence, these findings validated our analytical results.

\section{Discussion}

Recently, tumor immunotherapy has attracted an increasing amount of attention, and it is considered one of the most likely treatment methods to improve the survival rate of cancer patients. Immunotherapy has been successful in a variety of malignant tumors, including lung cancer, and several immunotherapy drugs have been approved by the Food and Drug Administration (FDA) [22, 23]. However, the application of immunotherapy in OS has been slow due to the complexity of the immune system and the subtle differences in the tumor-specific microenvironment as well as the fact that the tumor itself may resist immunotherapy in various ways [24]. It is necessary to determine predictive biomarkers with higher sensitivity and specificity to stratify patients to maximize the benefits for patients. In the TME, the interaction among tumor cells, immune cells and stromal cells promotes tumor proliferation and metastasis $[25,26]$. Therefore, a clear understanding of the complex TME and the complex immune characteristics of tumors and immune cells may be helpful to guide clinical immunotherapy.

The ESTIMATE and CIBERSORT algorithms are currently widely used to study the infiltration of immune cells and the proportion of cell subtypes in the TME. These two algorithms can transform the information of the gene expression matrix into an immune score and immune cell infiltration ratio. For instance, Lai et al. [27] studied the TME in renal cell carcinoma and identified effective biomarkers. Moreover, other tumor-related studies, such as lung cancer [28, 29] and liver cancer [30, 31], were also reported to use these algorithms. Interestingly, Hong et al. [32] performed a study on OS and found that SIGLEC7 and SP140 were related to OS prognosis. We will describe in more detail the differences between this study and another OS-related study. In summary, based on the above two algorithms, we can better understand the immune microenvironment information of OS, which is helpful to reveal the mechanism of tumor occurrence and discover effective biomarkers.

We searched the currently available public databases for OS, and the TARGET-OS and GSE39058 datasets were used in this study. Through the ESTIMATE algorithm, TARGET-OS showed that the immune score of the immune microenvironment of OS was closely related to prognosis; that is, the higher the immune score is, the better the prognosis. Although no statistically significant differences were found in GSE39058, it can be concluded from the survival curve that there was a significant difference between the high and low immune score groups. Therefore, we speculated that this might be caused by the insufficient number of patients. Then, we obtained the DEIGs by analyzing the difference between the high and low immune score groups and determined the intersection of DEIGs in the two datasets. The GO results revealed that these DEIGs were involved in various key immune responses, such as leukocyte proliferation, regulation of leukocyte differentiation, negative regulation of leukocyte activation, regulation of innate immune response and leukocyte-mediated cytotoxicity, while KEGG pathway analysis suggested that osteoclast differentiation, NK cell-mediated cytotoxicity and adaptive immune system 
were the most pivotal pathways affected by these genes. These items provide a direction for us to further study the mechanism of the immune response. Using univariate and multivariate regression analyses, we found that only MAP3K15 was statistically significant in both databases. MAP3K15, also known as ASK3, was reported to respond to osmotic stress in the kidney [33], and no research has been found to relate MAP3K15 to tumors. Thus, MAP3K15 may also play a significant role in tumor initiation and progression.

Subsequently, the CIBERSORT algorithm further identified the proportion of infiltrating immune cells in the TME of OS. We found that memory B cells, naive T cells, M2 macrophages and NK cell-activated cells were the main infiltrating cells. Then, we discussed the correlations between these immune cells and MAP3K15, as well as the correlations between these immune cells and the high- and low-risk groups. Finally, we found that MAP3K15 can well predict the prognosis of patients with OS, and MAP3K15 is closely related to CD4 T cells, M0 macrophages, and M1 macrophages; these results may provide new ideas for immunotherapy in the clinic.

In addition, we noticed that Hong et al. [32] also studied the microenvironment of OS. Although we both studied the TME of OS, we think that the two studies are different. In this research, we found a common DEIG (MAP3K15) by intersecting the analysis results, while in the study of Hong, they determined that SIGLEC7 and SP140 are DEIGS. This is reasonable because different research strategies were utilized. Moreover, we performed Cox regression analysis and analyzed the correlation of MAP3K15 and immune cells. In Hong's study, they did not further study the relationship between genes and immune cells.

Immune cell infiltration and the TME are hot research topics at present, and the development of bioinformatics methods has further improved our understanding of tumor molecular biology. However, there are still many limitations in this study. Although we validated the expression of MAP3K15 using qPCR assay and IHC and found that patients with high MAP3K15 expression had a significantly poorer prognosis, the correlation between immune infiltrated cells and MAP3K15 expression was not tested because of restrictions associated with our experimental conditions. Since we found a potential association between MAP3K15 expression and immunity, further mechanisms need to be explored.

Taken together, our study found that MAP3K15, whose expression level is closely related to immune activity in tumors, is a critical immune-related biomarker and may provide a basis for OS immunotherapy.

\section{Abbreviations}

OS Osteosarcoma

TARGET Therapeutically Applicable Research to Generate Effective Treatments GEO Gene Expression Omnibus

DEIGs Differentially expressed immune genes 
ROC Receiver operating characteristic

AUC Area under the curve

MAP3K15 Mitogen-activated protein kinase kinase kinase 15

TME The tumour microenvironment

PPI Protein-protein interaction

FPKM Fragments per kilobase of transcript per million mapped reads

GO Gene Ontology

KEGG Kyoto Encyclopedia of Genes and Genomes

MCODE Molecular complex detection

qPCR Quantitative polymerase chain reaction

IHC Immunohistochemistry

\section{Declarations}

\section{Consent for Publication}

Not applicable

\section{Availability of Data and Materials}

Data sharing not applicable to this article as no datasets were generated or analyzed during the current study.

\section{Competing Interests}

The authors declare that the research was conducted in the absence of any commercial or financial relationships that could be construed as a potential conflict of interest.

\section{Funding}

This study was financially supported by the National Natural Science Foundation of China (81871750, 81971518), the Key Realm R\&D Program of Guangdong Province (2019B020236001), and the Shenzhen Key Medical Discipline Construction Fund (ZDSYS20190902092851024). 


\section{Authors' Contributions}

ZC and HK performed the data analyses and wrote the manuscript. PW and HS helped perform the analysis with constructive discussions. All authors read and approved the final manuscript.

\section{Acknowledgements}

Not applicable

\section{References}

1. Siegel RL, Miller KD, Jemal A. Cancer statistics, 2018. CA Cancer J Clin. 2018;68:7-30.

2. Pingping B, Yuhong Z, Weiqi L, Chunxiao W, Chunfang W, Yuanjue S, et al. Incidence and mortality of sarcomas in Shanghai, China, during 2002-2014. Front Oncol. 2019;9:662.

3. Ritter J, Bielack SS. Osteosarcoma. Ann Oncol. 2010;21 Suppl 7:vii320-5.

4. Senerchia AA, Macedo CR, Ferman S, Scopinaro M, Cacciavillano W, Boldrini E, et al. Results of a randomized, prospective clinical trial evaluating metronomic chemotherapy in nonmetastatic patients with high-grade, operable osteosarcomas of the extremities: a report from the Latin American group of osteosarcoma treatment. Cancer. 2017;123:1003-10.

5. Bishop MW, Janeway KA, Gorlick R. Future directions in the treatment of osteosarcoma. Curr Opin Pediatr. 2016;28:26-33.

6. Dass CR, Choong PF. Gene therapy for osteosarcoma: steps towards clinical studies. J Pharm Pharmacol. 2008;60:405-13.

7. Yoshida K, Okamoto M, Aoki K, Takahashi J, Saito N. A review of T-cell related therapy for osteosarcoma. Int J Mol Sci. 2020;21:4877.

8. Yoshida K, Okamoto M, Sasaki J, Kuroda C, Ishida H, Ueda K, et al. Anti-PD-1 antibody decreases tumour-infiltrating regulatory T cells. BMC Cancer. 2020;20:25.

9. Thanindratarn P, Dean DC, Nelson SD, Hornicek FJ, Duan Z. Advances in immune checkpoint inhibitors for bone sarcoma therapy. J Bone Oncol. 2019;15:100221.

10. Le Cesne A, Marec-Berard P, Blay JY, Gaspar N, Bertucci F, Penel N, et al. Programmed cell death 1 (PD-1) targeting in patients with advanced osteosarcomas: results from the PEMBROSARC study. Eur J Cancer. 2019;119:151-7.

11. Zheng B, Ren T, Huang Y, Sun K, Wang S, Bao X, et al. PD-1 axis expression in musculoskeletal tumors and antitumor effect of nivolumab in osteosarcoma model of humanized mouse. J Hematol Oncol. 2018;11:16.

12. Wu T, Dai Y. Tumor microenvironment and therapeutic response. Cancer Lett. 2017;387:61-8.

13. Locy H, De Mey S, De Mey W, De Ridder M, Thielemans K, Maenhout SK. Immunomodulation of the tumor microenvironment: turn foe into friend. Front Immunol. 2018;9:2909. 
14. Cersosimo F, Lonardi S, Bernardini G, Telfer B, Mandelli GE, Santucci A, et al. Tumor-associated macrophages in osteosarcoma: from mechanisms to therapy. Int J Mol Sci. 2020;21:5207.

15. Yoshihara K, Shahmoradgoli M, Martínez E, Vegesna R, Kim H, Torres-Garcia W, et al. Inferring tumour purity and stromal and immune cell admixture from expression data. Nat Commun. 2013;4:2612.

16. Newman AM, Liu CL, Green MR, Gentles AJ, Feng W, Xu Y, et al. Robust enumeration of cell subsets from tissue expression profiles. Nat Methods. 2015;12:453-7.

17. Gentles AJ, Newman AM, Liu CL, Bratman SV, Feng W, Kim D, et al. The prognostic landscape of genes and infiltrating immune cells across human cancers. Nat Med. 2015;21:938-45.

18. Mao S, Li Y, Lu Z, Che Y, Huang J, Lei Y, et al. Systematic profiling of immune signatures identifies prognostic predictors in lung adenocarcinoma. Cell Oncol (Dordr). 2020;43:681-94.

19. Deng L, Lu D, Bai Y, Wang Y, Bu H, Zheng H. Immune profiles of tumor microenvironment and clinical prognosis among women with triple-negative breast cancer. Cancer Epidemiol Biomarkers Prev. 2019;28:1977-85.

20. Liu F, Qin L, Liao Z, Song J, Yuan C, Liu Y, et al. Microenvironment characterization and multi-omics signatures related to prognosis and immunotherapy response of hepatocellular carcinoma. Exp Hematol Oncol. 2020;9:10.

21. Law CW, Alhamdoosh M, Su S, Dong X, Tian L, Smyth GK, et al. RNA-seq analysis is easy as 1-2-3 with limma, Glimma and edgeR. F1000Res. 2016;5:ISCB Comm J-1408.

22. Subbiah V, Solit DB, Chan TA, Kurzrock R. The FDA approval of pembrolizumab for adult and pediatric patients with tumor mutational burden $(T M B) \geq 10$ : a decision centered on empowering patients and their physicians. Ann Oncol. 2020;31:1115-8.

23. Wu Y, Mou H. Complete remission in a patient with advanced renal pelvis carcinoma with lung metastasis treated with durvalumab immunotherapy: a case report. Ann Palliat Med. 2020;9:240813.

24. Pratt HG, Justin EM, Lindsey BA. Applying osteosarcoma immunology to understand disease progression and assess immunotherapeutic response. Adv Exp Med Biol. 2020;1258:91-109.

25. Cao R, Yuan L, Ma B, Wang G, Tian Y. Tumour microenvironment (TME) characterization identified prognosis and immunotherapy response in muscle-invasive bladder cancer (MIBC). Cancer Immunol Immunother. 2020;70:1-18.

26. Giraldo NA, Sanchez-Salas R, Peske JD, Vano Y, Becht E, Petitprez F, et al. The clinical role of the TME in solid cancer. Br J Cancer. 2019;120:45-53.

27. Lai $Y$, Tang F, Huang $Y, H e ~ C$, Chen $C$, Zhao J, et al. The tumour microenvironment and metabolism in renal cell carcinoma targeted or immune therapy. J Cell Physiol. 2020;236:1616-27.

28. Zhang L, Chen J, Cheng T, Yang H, Li H, Pan C. Identification of the key genes and characterizations of Tumor Immune Microenvironment in Lung Adenocarcinoma (LUAD) and Lung Squamous Cell Carcinoma (LUSC). J Cancer. 2020;11:4965-79. 
29. Liu Z, Wan Y, Qiu Y, Qi X, Yang M, Huang J, et al. Development and validation of a novel immunerelated prognostic model in lung squamous cell carcinoma. Int J Med Sci. 2020;17:1393-405.

30. Liu X, Niu X, Qiu Z. A five-gene signature based on stromal/immune scores in the tumor microenvironment and its clinical implications for liver cancer. DNA Cell Biol. 2020;39:1621-38.

31. Zhang FP, Huang YP, Luo WX, Deng WY, Liu CQ, Xu LB, et al. Construction of a risk score prognosis model based on hepatocellular carcinoma microenvironment. World J Gastroenterol. 2020;26:13453.

32. Hong W, Yuan H, Gu Y, Liu M, Ji Y, Huang Z, et al. Immune-related prognosis biomarkers associated with osteosarcoma microenvironment. Cancer Cell Int. 2020;20:83.

33. Naguro I, Umeda T, Kobayashi Y, Maruyama J, Hattori K, Shimizu Y, et al. ASK3 responds to osmotic stress and regulates blood pressure by suppressing WNK1-SPAK/OSR1 signaling in the kidney. Nat Commun. 2012;3:1285.

\section{Figures}

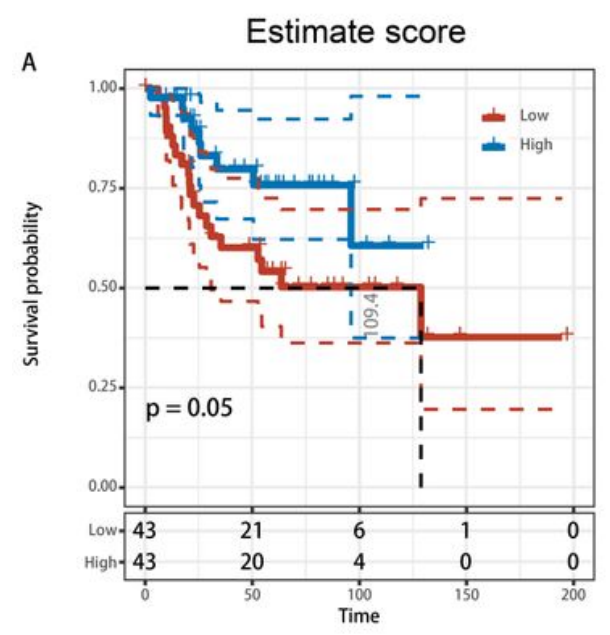

Estimate score

D

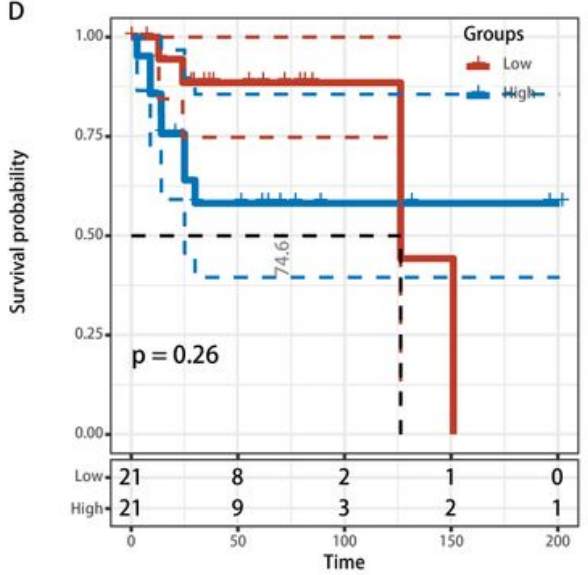

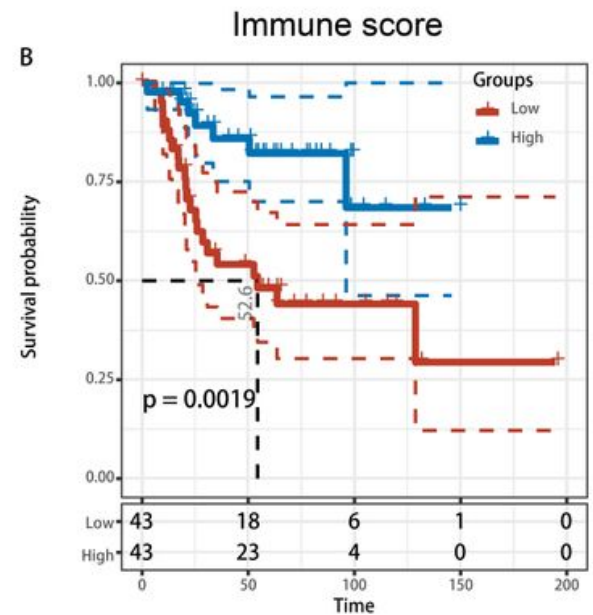

Immune score

$E$

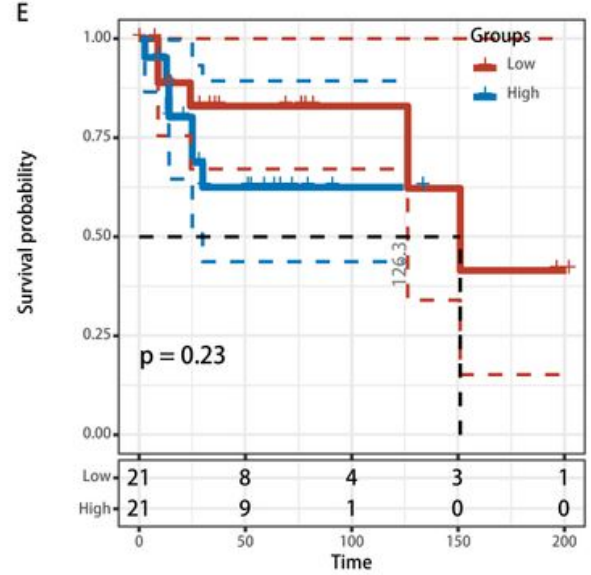

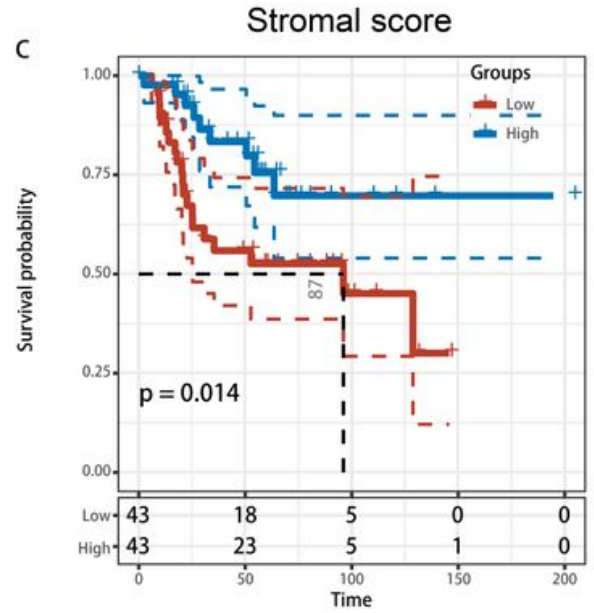

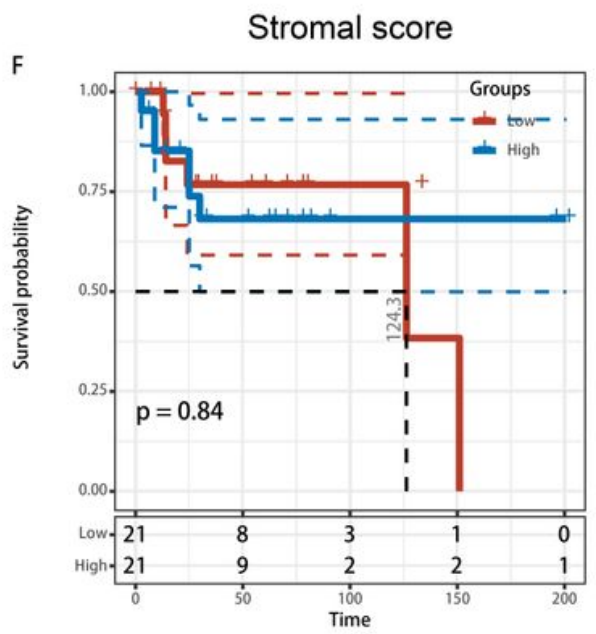

Figure 1 
The prognostic significance of the estimated immune and stromal scores in the TARGET-OS and GSE39058 datasets. (a) Overall survival according to the estimated score in the TARGET-OS dataset. (b) Overall survival according to the immune score in the TARGET-OS dataset. (c) Overall survival according to the stromal score in the TARGET-OS dataset. (d) Overall survival according to the estimated score in the GSE39058 dataset. (e) Overall survival according to the immune score in the GSE39058 dataset. (f) Overall survival according to the stromal score in the GSE39058 dataset.

A

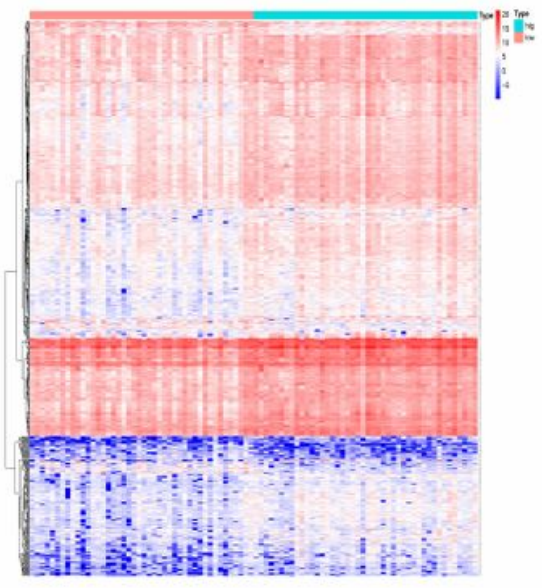

B

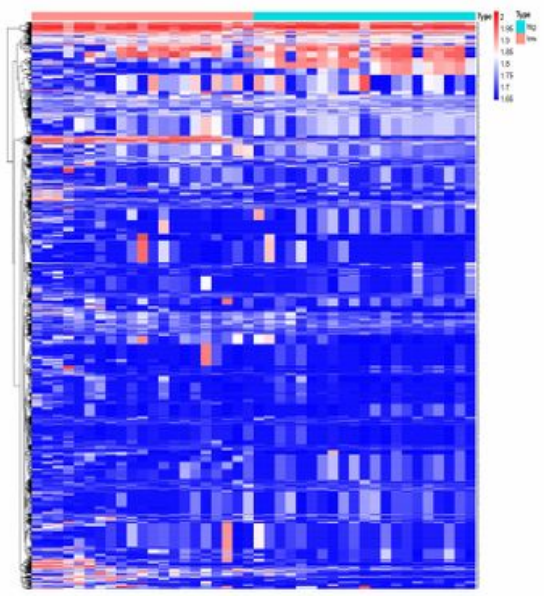

C

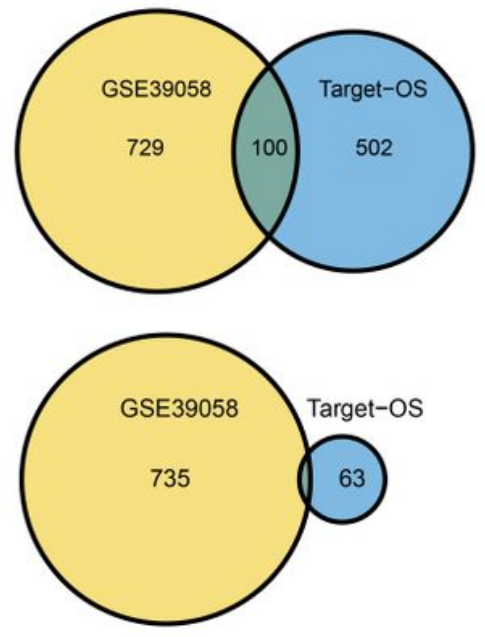

$\mathrm{F}$

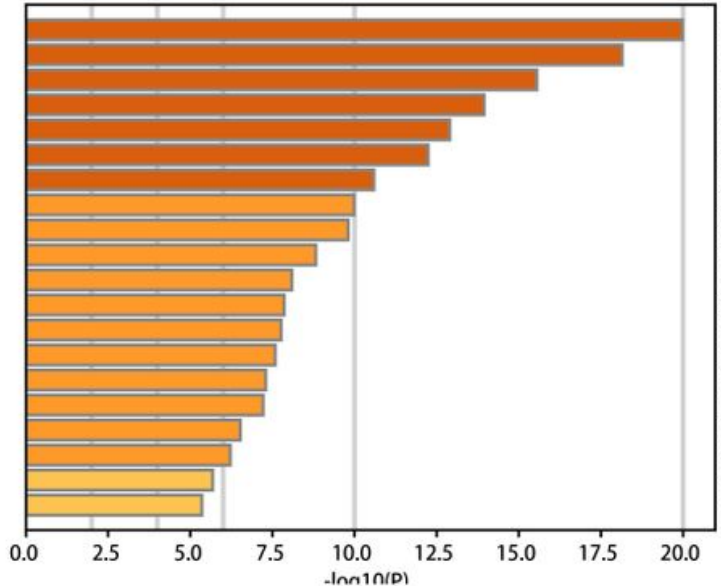

GO:0070661: leukocyte proliferation GO:0019221: cytokine-mediated signaling pathway R-HSA-1280218: Adaptive Immune System GO:0002274: myeloid leukocyte activation GO:1902105: regulation of leukocyte differentiation hsa04380: Osteoclast differentiation GO:0002695: negative regulation of leukocyte activation GO:0006909: phagocytosis

GO:0045088: regulation of innate immune response GO:0001909: leukocyte mediated cytotoxicity hsa04650: Natural killer cell mediated cytotoxicity GO:0032623: interleukin-2 production R-HSA-449147: Signaling by Interleukins GO:0007229: integrin-mediated signaling pathway GO:0070374: positive regulation of ERK1 and ERK2 cascade GO:0098542: defense response to other organism GO:0043900: regulation of multi-organism process GO:0045730: respiratory burst GO:0050663: cytokine secretion GO:0042116: macrophage activation

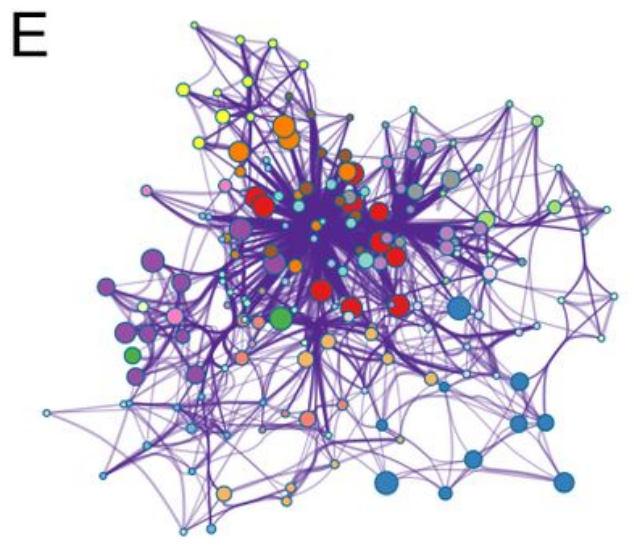

leukocyte proliferation cytokine-mediated signaling pathway Adaptive Immune System myeloid leukocyte activation regulation of leukocyte differentiation Osteoclast differentiation

negative regulation of leukocyte activation phagocytosis

regulation of innate immune response

leukocyte mediated cytotoxicity

Natural killer cell mediated cytotoxicity

interleukin-2 production

- Signaling by Interleukins

integrin-mediated signaling pathway

positive regulation of ERK1 and ERK2 cascade

defense response to other organism

regulation of multi-organism process

respiratory burst

cytokine secretion

macrophage activation
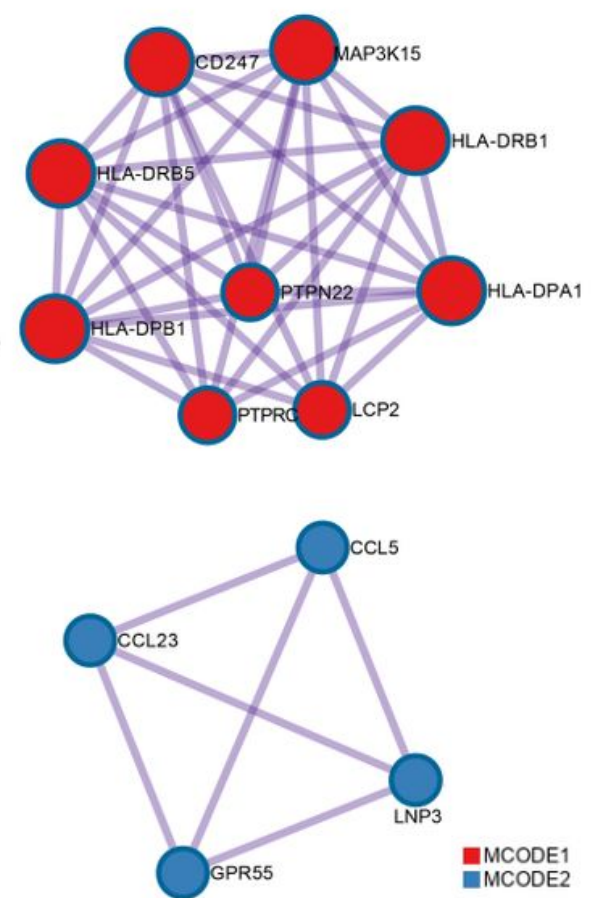

Figure 2 
Overview of the DEIGs in OS. (a) A heatmap of the expression profile in the TARGET-OS dataset. (b) A heatmap of the expression profile in the GSE39058 dataset. (c) A Venn diagram shows the common DEIGs in both datasets. (d) Bar plots displaying GO and KEGG analysis results based on Metascape. (e) Network display GO and KEGG analysis results based on Metascape. (f) MCODE results obtained from the PPI network.

A
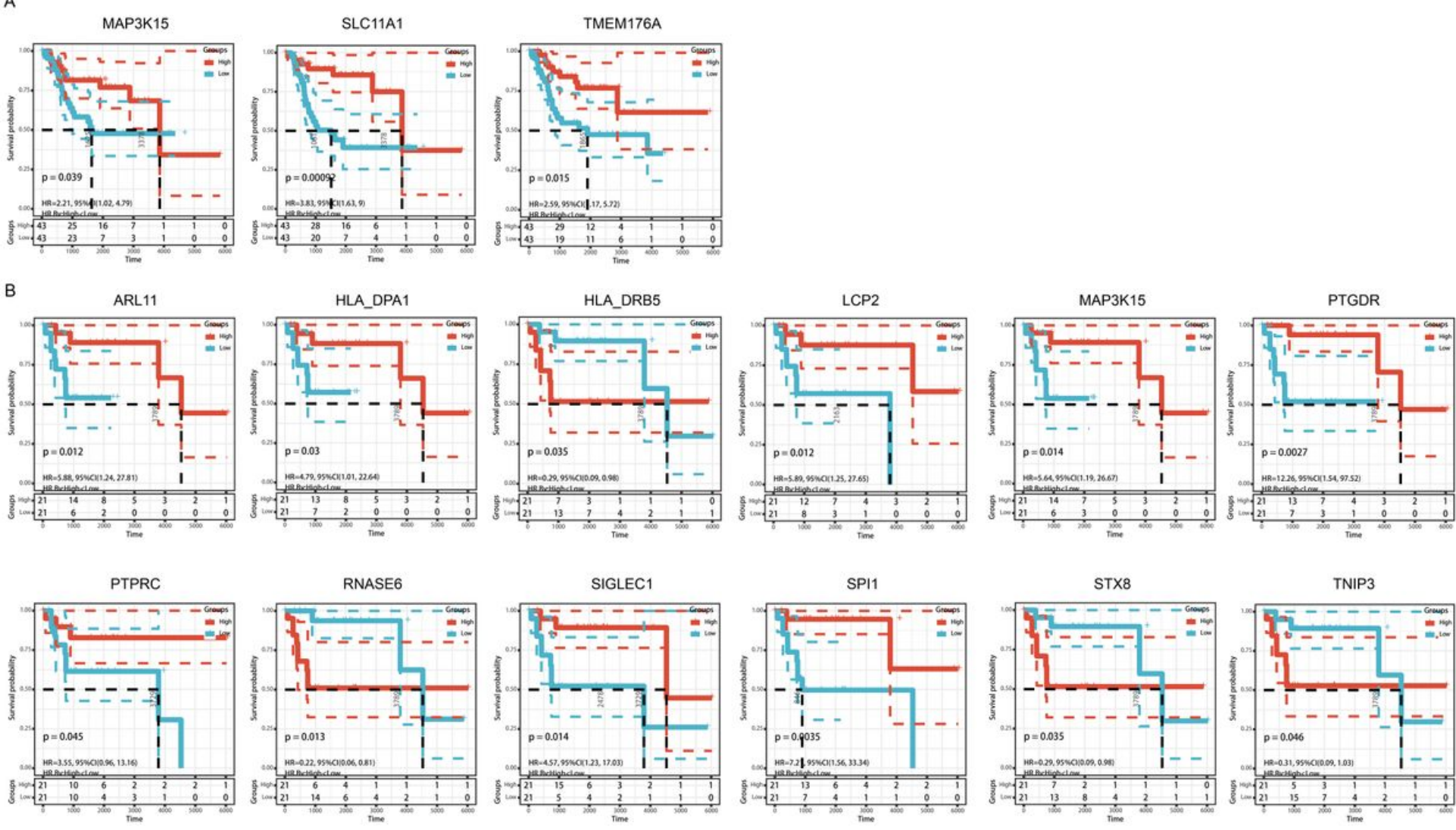

\section{Figure 3}

Survival curves of the DEIGs were created using the Kaplan-Meier curve in the SangerBox database. (a) DEIGs with prognostic values in the TARGET-OS dataset. (b) DEIGs with prognostic value in the GSE39058 dataset. 

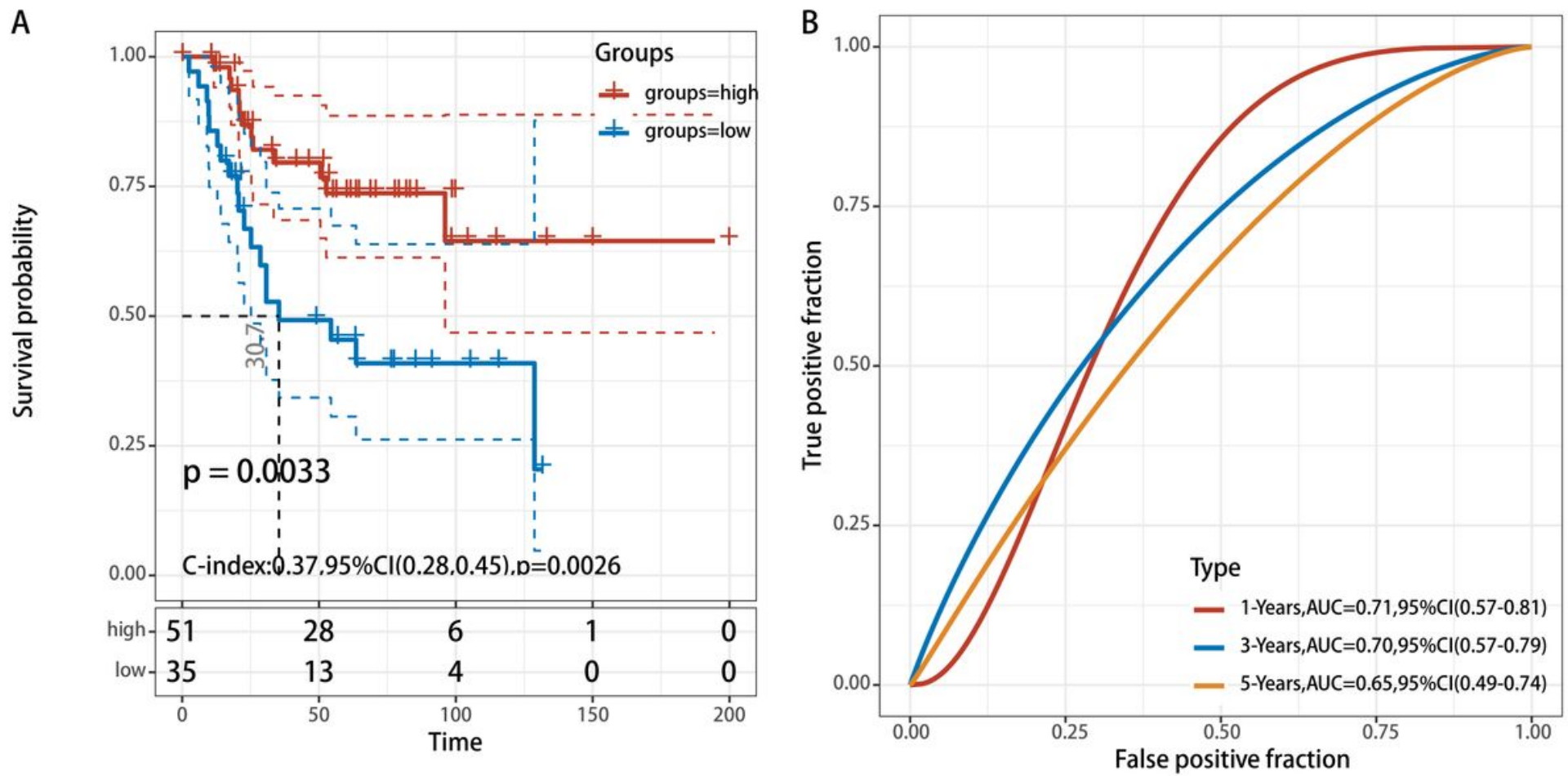

Figure 4

Kaplan-Meier plot of MAP3K15 and ROC curve of the prognostic immune signature. (a) The predictive immune model constructed from MAP3K15. (b) ROC curves of the immune signature with the corresponding AUCs indicated on the side. 
A

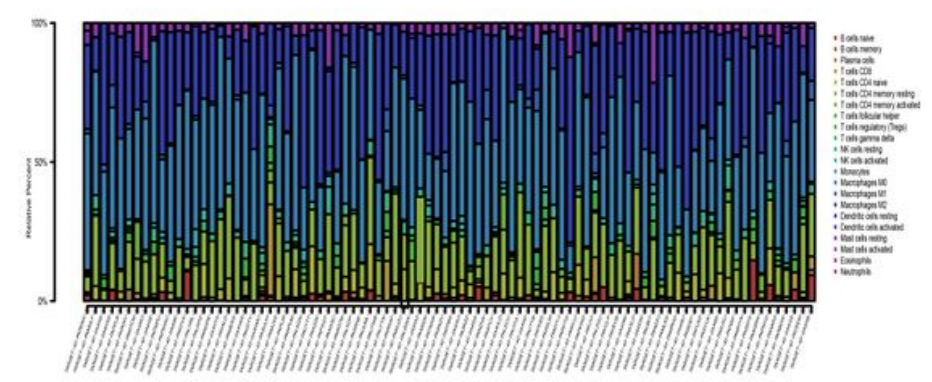

C

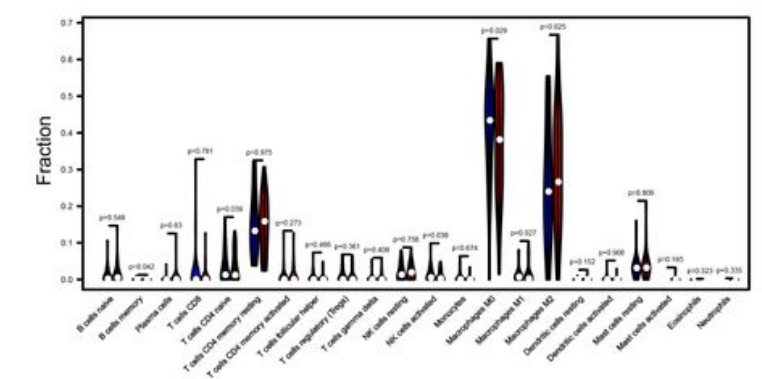

E

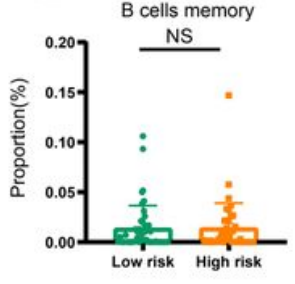

Macrophages MO

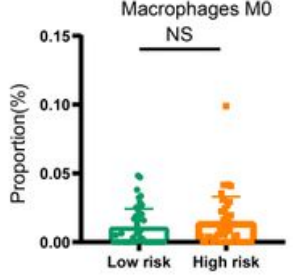

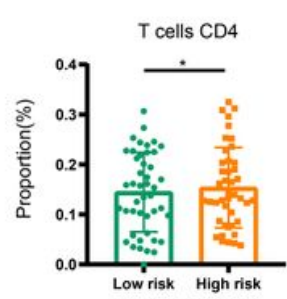

Macrophages M1

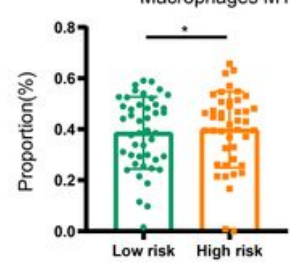

B

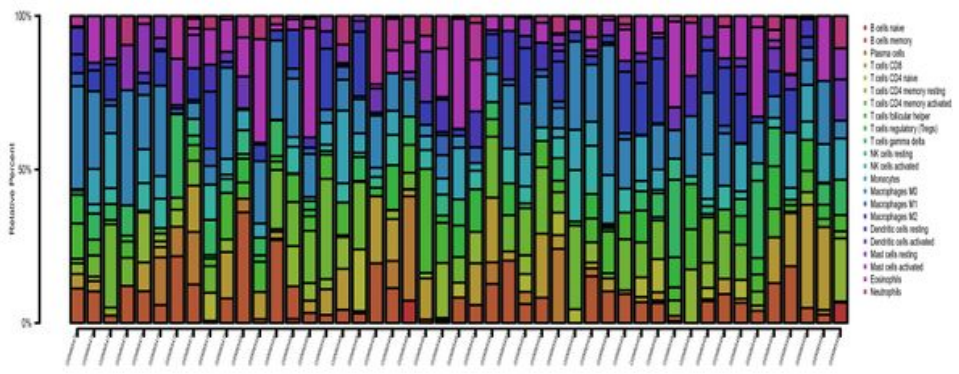

D

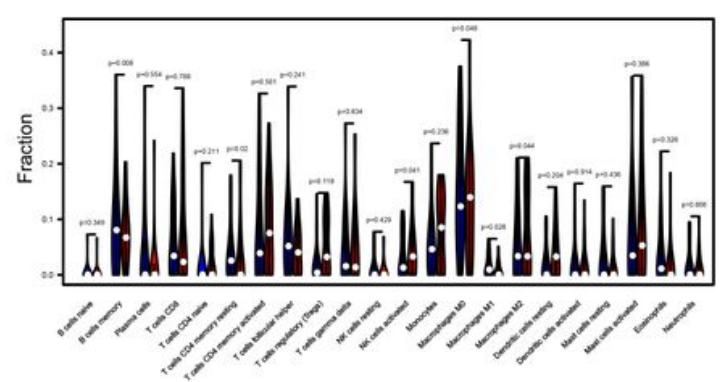

F
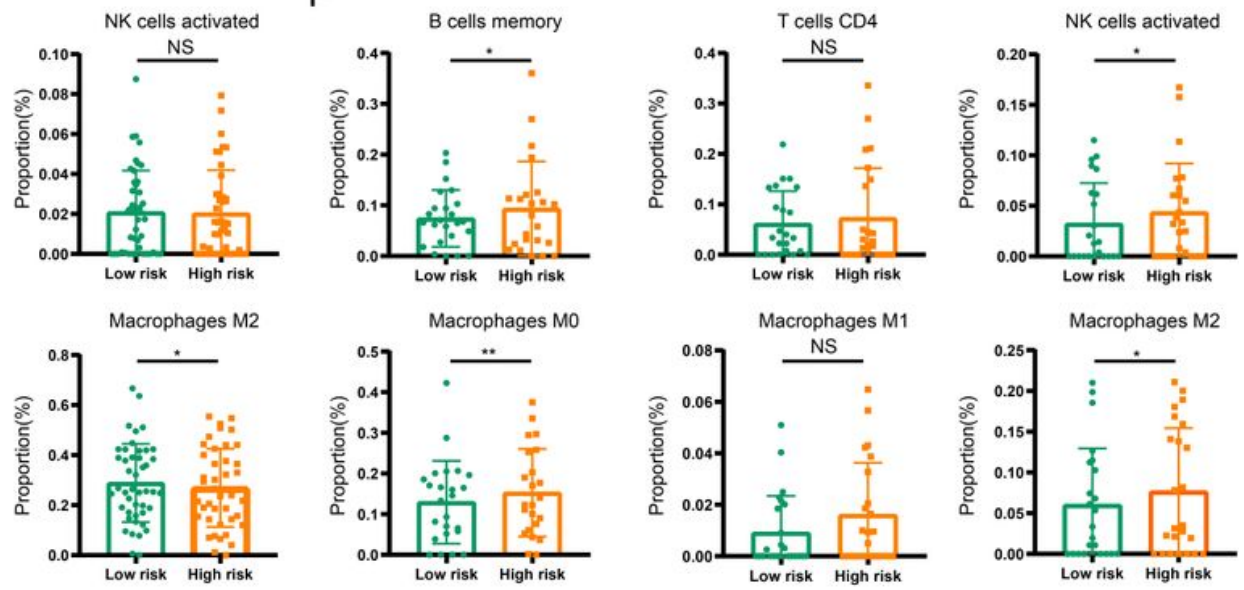

Figure 5

Immune cell landscapes in different immune score groups. (a) The proportion of immune cells in the Target-OS dataset. (b) The proportion of immune cells in the GSE39058 dataset. (c) High immune score and low immune score groups in the TARGET-OS dataset. (d) High immune score and low immune score groups in the GSE39058 dataset. (e) Proportions of memory B cells, CD4 T cells, activated NK cells, M0 macrophages, M1 macrophages, and M2 macrophages in different immune score groups (TARGET-OS). (f) Proportions of memory B cells, CD4 T cells, activated NK cells, M0 macrophages, M1 macrophages, and M2 macrophages in different immune score groups (GSE39058). 

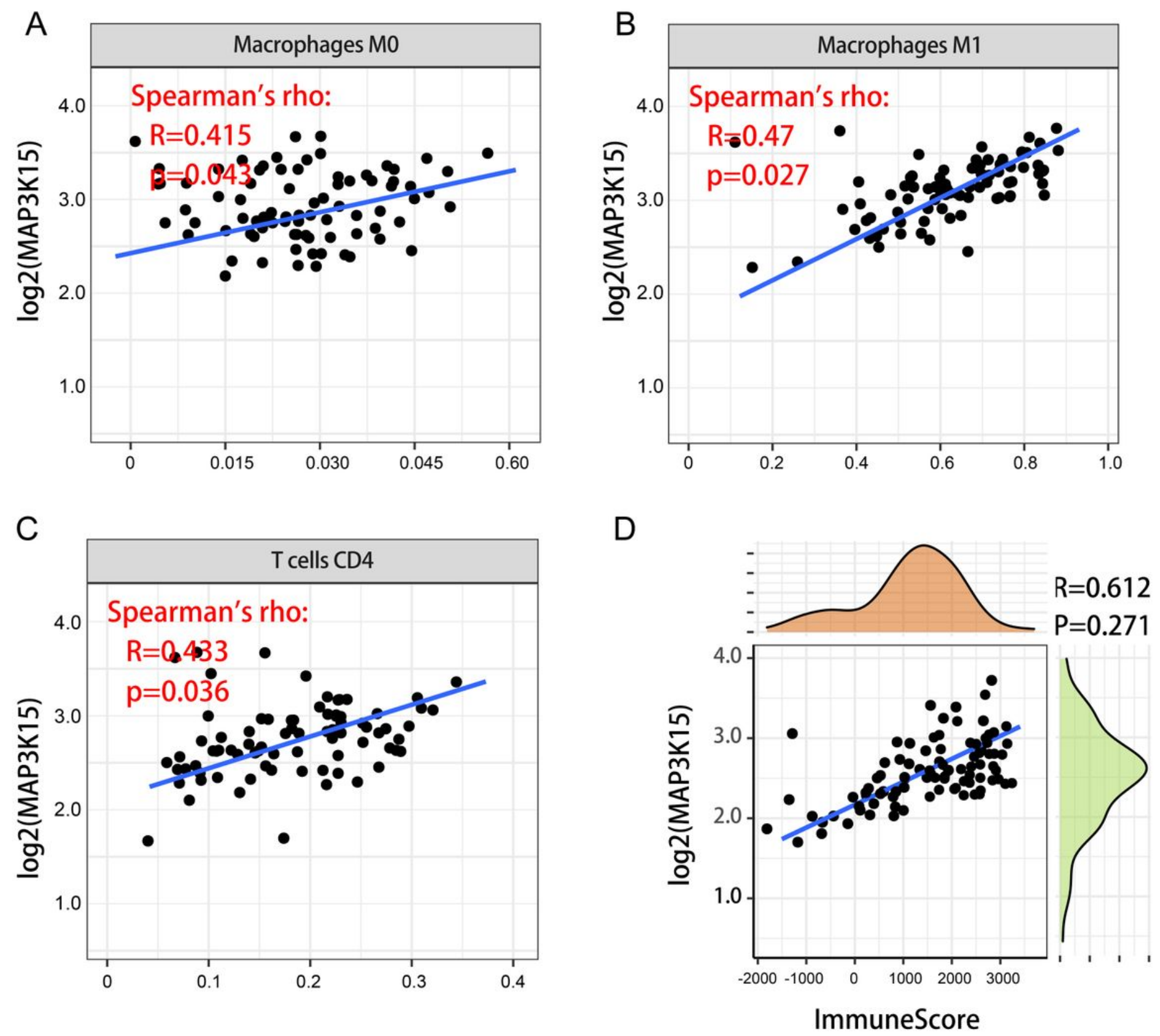

Figure 6

Correlations between prognostic signature and infiltrating immune cells. (a)-(c) The expression of MAP3K15 and CD4 T cells, M0 macrophages, and M1 macrophages was positively correlated. (d) MAP3K15 was positively correlated with the immune score. 
A

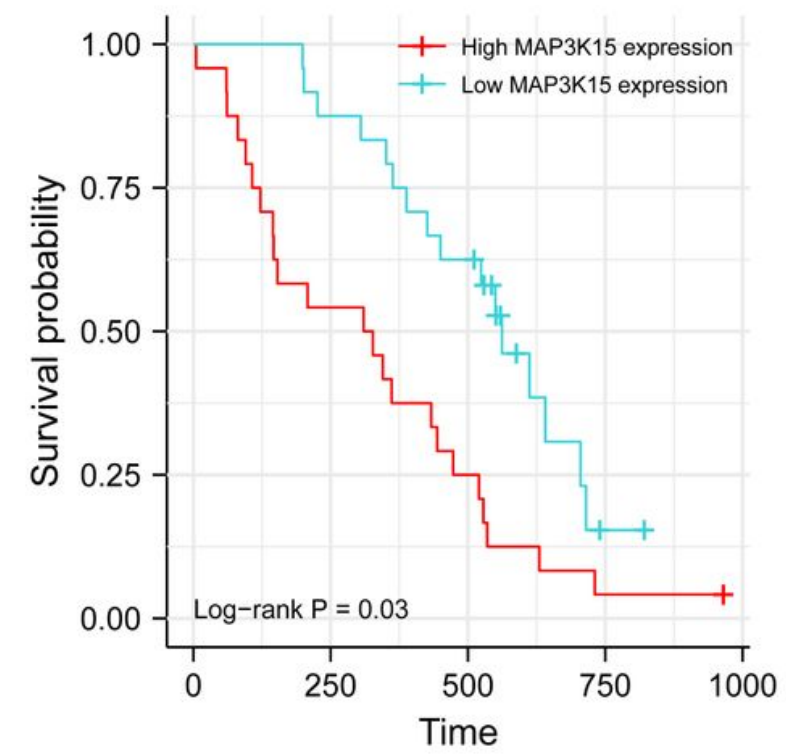

B
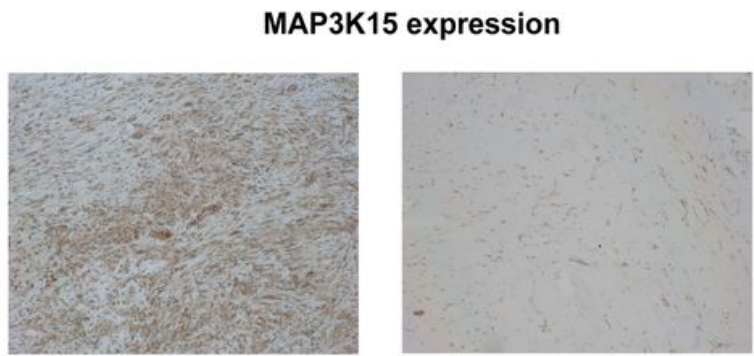

$\mathbf{X 2 0 0}$

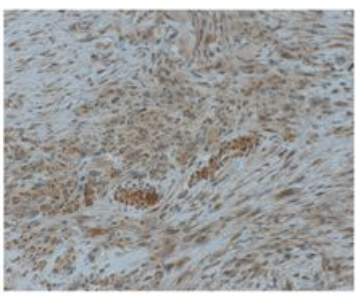

High group

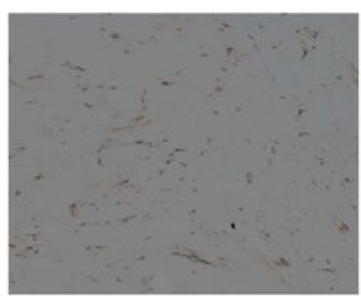

Low group

\section{Figure 7}

MAP3K15 expression validation and prognosis analysis. (a) The OS rates of patients with high and low expression of MAP3K15 were evaluated by the log-rank method. (b) Representative histopathologic images of MAP3K15 expression in osteosarcoma.

\section{Supplementary Files}

This is a list of supplementary files associated with this preprint. Click to download.

- FigS1.jpg 